\title{
L-Stable Derivative-Free Error-Corrected Trapezoidal Rule for Burgers' Equation with Inconsistent Initial and Boundary Conditions
}

\author{
Lajja Verma \\ Department of Mathematics, BITS Pilani, Rajasthan, Pilani 333031, India \\ Correspondence should be addressed to Lajja Verma, lajjamaths@yahoo.co.in
}

Received 25 March 2012; Revised 19 August 2012; Accepted 21 August 2012

Academic Editor: Theodore E. Simos

Copyright (C 2012 Lajja Verma. This is an open access article distributed under the Creative Commons Attribution License, which permits unrestricted use, distribution, and reproduction in any medium, provided the original work is properly cited.

We combine suitable arithmetic average approximations, with explicit backward Euler formula, and derive a third-order $L$-stable derivative-free error-corrected trapezoidal rule (LSDFECT). Then, we apply LSDFECT rule to the linearized Burgers' equation with inconsistent initial and boundary conditions and test its stability and exactness. We use Mathematica 7.0 for computation.

\section{Introduction}

Nonlinear phenomena play a crucial role in applied mathematics and physics and in mechanics and biology. Burgers' equation has been found to describe various kinds of phenomena such as a mathematical model of turbulence and the approximate theory of flow through a shock wave traveling in a viscous fluid. The equation has found applications in field as diverse as number theory, gas dynamics, heat conduction, and elasticity. In past years, a growing interest has been given to the propagation of nonlinear waves in nonlinear dynamical systems. A nonlinear wave is one of the fundamental objects of nature. These waves appear in a great array of contexts such as hydrodynamics, nonlinear optics, plasmas, solid-state physics, nuclear physics, and many other nonlinear phenomena. The systems are often described by nonlinear partial differential equations with constant or variable coefficients. In this paper, we are proposing a method which can deal efficiently with numerical solutions of Burgers' equation which contains inconsistencies in terms of initial and boundary conditions.

$L$-stable numerical methods are sought whenever inconsistencies are present in the initial and boundary conditions or in some other cases. So, in the present paper, we use derivative-free error-corrected trapezoidal rule [1], arithmetic average approximation, and 
explicit Euler's backward formula on $y^{\prime}=f(t, y)$ and develop $L$-stable derivative-free errorcorrected trapezoidal type quadrature formula.

We use these results to study the numerical solution of one-dimensional quasilinear partial differential equation, that is, Burgers' equation

$$
\frac{\partial w}{\partial t}+w \frac{\partial w}{\partial x}-\frac{v_{d}}{2} \frac{\partial^{2} w}{\partial x^{2}}=0, \quad(x, t) \in[0,1] \times[0, T],
$$

with initial condition

$$
w(x, 0)=g(x), \quad 0<x<1,
$$

and Dirichlet boundary conditions

$$
w(0, t)=0=w(1, t), \quad 0 \leq t \leq T,
$$

where $v_{d}>0$ is a coefficient of viscous diffusion and $g$ is sufficiently smooth given function.

We consider some examples of Burgers' equation with Dirichlet boundary condition and transform it into linear diffusion equation with Neumann boundary condition for which $\mathrm{CN}$ produces unacceptable oscillations while the present $L$-stable derivative-free errorcorrected trapezoidal type rule provides both stable and accurate result.

The Crank and Nicolson [2] scheme is $A$-stable not $L$-stable and produces undesirable oscillations in the case of inconsistencies in the initial and boundary conditions [3] or when time step taken is large [4]. As an alternative to $\mathrm{CN}$, Chawla et al. [5] have proposed generalized trapezoidal formula $(\operatorname{GTF}(\alpha))$ which is second-order $L$-stable schemes for $\alpha>0$ and gives quite stable and accurate approximations for a judicious choice of the parameter $\alpha$.

For higher-order accuracy, Chawla et al. [6] proposed a modified $A$-stable Simpson's $1 / 3$ rule (ASIMP) but, since it lacks L-stability, ASIMP also produces unwanted oscillations. To rectify this, Chawla and Evans [7] and Pandey et al. [8] presented a $L$-stable version of the Simpson's 1/3 rule and Simpson's 3/8 rule, respectively, and employed it to derive a thirdorder time integration scheme for the diffusion equation. These $L$-stable schemes provide stable and accurate approximations.

This paper is organized in 4 sections. In Section 2, we discuss derivative-free errorcorrected trapezoidal rule. In Section 3 we derive $L$-stable version of derivative free error corrected trapezoidal rule and discuss its local truncation error and stability and apply it to linearized Burgers' equation. Finally, in Section 4, we illustrate our findings numerically.

\section{Derivative-Free Error-Corrected Trapezoidal Rule}

Let $f(x)$ be defined over the interval $[a, b]$. Let us divide the interval $[a, b]$ into $n$ subintervals $\left[x_{i-1}, x_{i}\right]$ for $i=1(1) n$, where $x_{i}=x_{0}+i * h, x_{0}=a$ and $x_{n}=b$. Then

$$
\begin{gathered}
\int_{a}^{b} f(x) d x=\sum_{i=1}^{n} \operatorname{Trap}\left[x_{i-1}, x_{i}\right], \\
\int_{a}^{b} f(x) d x=\frac{1}{2}\left\{\sum_{i=1}^{n-1} \operatorname{Simp}_{1 / 3}\left[x_{i-1}, x_{i+1}\right]+\operatorname{Simp}_{1 / 3}\left[x_{0}, x_{1}\right]+\operatorname{Simp}_{1 / 3}\left[x_{n-1}, x_{n}\right]\right\},
\end{gathered}
$$


where

$$
\begin{gathered}
\operatorname{Trap}\left[x_{i-1}, x_{i}\right]=\frac{h}{2}\left[f\left(x_{i-1}\right)+f\left(x_{i}\right)\right], \\
\operatorname{Simp}_{1 / 3}\left[x_{i-1}, x_{i+1}\right]=\frac{h}{3}\left[f\left(x_{i-1}\right)+4 f\left(x_{i}\right)+f\left(x_{i+1}\right)\right] .
\end{gathered}
$$

Now derivative-free corrector is the difference between (2.1) and is given by (cf. [1])

$$
-\frac{h}{4}\left(f_{0}+f_{n}\right)+\frac{h}{3}\left(f_{1 / 2}+f_{n-(1 / 2)}\right)-\frac{h}{12}\left(f_{1}+f_{n-1}\right) .
$$

Hence derivative-free error-corrected trapezoidal rule is given by

$$
h\left[\frac{1}{2} f_{0}+f_{1}+f_{2}+\cdots+f_{n-1}+\frac{1}{2} f_{n}\right]-\frac{h}{4}\left(f_{0}+f_{n}\right)+\frac{h}{3}\left(f_{1 / 2}+f_{n-(1 / 2)}\right)-\frac{h}{12}\left(f_{1}+f_{n-1}\right) .
$$

The above quadrature formula will have error proportional to $(b-a) h^{4}$, and it does not contain any derivative term.

\section{L-Stable Derivative-Free Error-Corrected Trapezoidal-Type Rule}

Definition 3.1. A method is said to be $L$-stable if it is $A$-stable, and, in addition, when applied to the scalar test equation $y^{\prime}=\lambda y, \lambda \in \mathbb{C}, \operatorname{Re}(\lambda)<0$, it yields $y_{n+1}=\Psi(z) y_{n}, z=\lambda h$ ( $h$ is step size), where $|\Psi(z)| \rightarrow 0$ as $\operatorname{Re}(z) \rightarrow-\infty$.

Consider the first-order initial value problem

$$
y^{\prime}(t)=f(t, y), \quad y\left(t_{0}\right)=\eta \text {. }
$$

The derivative-free error-corrected trapezoidal-type rule is given by

$$
y_{n+1}=y_{n}+\frac{h}{36}\left[3\left(f_{n}+f_{n+1}\right)+11\left(f_{n+(1 / 3)}+f_{n+(2 / 3)}\right)+4\left(f_{n+(1 / 6)}+f_{n+(5 / 6)}\right)\right] \text {. }
$$

We start with following suitable weighted average approximations

$$
\begin{aligned}
& y_{n+1 / 3}=\frac{2 y_{n}+y_{n+1}}{3}, \\
& y_{n+2 / 3}=\frac{y_{n}+2 y_{n+1}}{3}, \\
& y_{n+1 / 6}=\frac{5 y_{n}+y_{n+1}}{6}, \\
& y_{n+5 / 6}=\frac{y_{n}+5 y_{n+1}}{6} .
\end{aligned}
$$


The explicit backward Euler formula (Chawla et al. [9]) is

$$
y_{n}=y_{n+1}-h f_{n+1} .
$$

Now we use (3.3) to obtain the following approximations:

$$
\begin{gathered}
\bar{y}_{n+1 / 3}=\frac{4 y_{n}+5 y_{n+1}}{9}-\frac{2}{9} h f_{n+1}, \\
\bar{y}_{n+2 / 3}=\frac{y_{n}+8 y_{n+1}}{9}-\frac{2}{9} h f_{n+1}, \\
\bar{y}_{n+1 / 6}=\frac{23 y_{n}+13 y_{n+1}}{36}-\frac{7}{36} h f_{n+1}, \\
\bar{y}_{n+5 / 6}=\frac{y_{n}+11 y_{n+1}}{12}-\frac{1}{12} h f_{n+1} .
\end{gathered}
$$

Define $\bar{f}_{i}=f\left(x_{i}, \bar{y}_{i}\right)$ and use it to derive our new LSDFECT rule which is given by

$$
y_{n+1}=y_{n}+\frac{h}{36}\left[3\left(f_{n}+f_{n+1}\right)+11\left(\bar{f}_{n+1 / 3}+\bar{f}_{n+2 / 3}\right)+4\left(\bar{f}_{n+1 / 6}+\bar{f}_{n+5 / 6}\right)\right] .
$$

\subsection{Local Truncation Error}

Since

$$
\begin{gathered}
y_{n+1 / 3}=\frac{2 y_{n}+y_{n+1}}{3}-\frac{1}{9} h^{2} y_{n}^{\prime \prime}-\frac{4}{81} h^{3} y_{n}^{\prime \prime \prime}-\frac{13}{324} h^{4} y_{n}^{(4)}+O\left(h^{5}\right), \\
y_{n+2 / 3}=\frac{y_{n}+2 y_{n+1}}{3}-\frac{1}{9} h^{2} y_{n}^{\prime \prime}-\frac{5}{81} h^{3} y_{n}^{\prime \prime \prime}-\frac{19}{972} h^{4} y_{n}^{(4)}+O\left(h^{5}\right), \\
y_{n+1 / 6}=\frac{5 y_{n}+y_{n+1}}{6}-\frac{5}{72} h^{2} y_{n}^{\prime \prime}-\frac{35}{1296} h^{3} y_{n}^{\prime \prime \prime}-\frac{215}{31104} h^{4} y_{n}^{(4)}+O\left(h^{5}\right), \\
y_{n+5 / 6}=\frac{y_{n}+5 y_{n+1}}{6}-\frac{5}{72} h^{2} y_{n}^{\prime \prime}-\frac{55}{1296} h^{3} y_{n}^{\prime \prime \prime}-\frac{455}{31104} h^{4} y_{n}^{(4)}+O\left(h^{5}\right), \\
y_{n}=y_{n+1}-h f_{n+1}+\frac{1}{2} h^{2} y_{n}^{\prime \prime}+\frac{1}{3} h^{3} y_{n}^{\prime \prime \prime}+\frac{1}{8} h^{4} y_{n}^{(4)}+O\left(h^{5}\right) .
\end{gathered}
$$


We deduce that

$$
\begin{aligned}
& \bar{y}_{n+1 / 3}=y_{n+1 / 3}-\frac{2}{81} h^{3} y_{n}^{\prime \prime \prime}+\frac{1}{81} h^{4} y_{n}^{(4)}+O\left(h^{5}\right) \\
& \bar{y}_{n+2 / 3}=y_{n+2 / 3}-\frac{1}{81} h^{3} y_{n}^{\prime \prime \prime}-\frac{2}{243} h^{4} y_{n}^{(4)}+O\left(h^{5}\right) \\
& \bar{y}_{n+1 / 6}=y_{n+1 / 6}-\frac{1}{36} h^{2} y_{n}^{\prime \prime}+\frac{23}{1296} h^{3} y_{n}^{(3)}+O\left(h^{4}\right) \\
& \bar{y}_{n+5 / 6}=y_{n+5 / 6}+\frac{1}{36} h^{2} y_{n}^{\prime \prime \prime}-\frac{19}{1296} h^{3} y_{n}^{(3)}+O\left(h^{4}\right)
\end{aligned}
$$

Again since

$$
y_{n+1}=y_{n}+\frac{h}{36}\left[3\left(f_{n}+f_{n+1}\right)+11\left(f_{n+1 / 3}+f_{n+2 / 3}\right)+4\left(f_{n+1 / 6}+f_{n+5 / 6}\right)\right]+O\left(h^{5}\right),
$$

it can be shown that

$$
y_{n+1}=y_{n}+\frac{h}{36}\left[3\left(f_{n}+f_{n+1}\right)+11\left(\bar{f}_{n+1 / 3}+\bar{f}_{n+2 / 3}\right)+4\left(\bar{f}_{n+1 / 6}+\bar{f}_{n+5 / 6}\right)\right]+t_{n}(h)
$$

where

$$
t_{n}(h)=O\left(h^{4}\right)
$$

Thus scheme is of third order.

\subsection{Stability}

Applying scheme (3.6) to the test equation

$$
y^{\prime}=-\lambda y, \quad \lambda>0,
$$

and setting $z=\lambda h$, we have

$$
y_{n+1}=\Psi(z) y_{n}
$$

where $\Psi(z)=\left((1-(1 / 3) z) /\left(1+(2 / 3) z+(1 / 6) z^{2}\right)\right)$. Since $\Psi(z) \rightarrow 0$ as $z \rightarrow \infty$, the scheme (3.6) is $L$-stable.

Therefore, it follows that time integration scheme (3.6) is of third order and $L$-stable. We call it LSDFECT rule for the first-order initial value problem. 


\section{LSDFECT Rule for Partial Differential Equation}

\subsection{The Hopf and Cole Transformation}

Hopf [10] and Cole [11] suggested that (1.1) can be reduced to linear heat equation by a nonlinear transformation given by

$$
\begin{gathered}
\psi=-v_{d}(\log \phi), \\
w=\psi_{x} .
\end{gathered}
$$

Using (4.1) in (1.1), we have

$$
\begin{gathered}
\frac{\partial \phi}{\partial t}=\frac{v_{d}}{2} \frac{\partial^{2} \phi}{\partial x^{2}}, \\
\phi_{x}(0, t)=\phi_{x}(1, t)=0, \\
\phi(x, 0)=f(x) .
\end{gathered}
$$

The Fourier series solution to the linearized heat equation (4.2) is

$$
\phi(x, t)=A_{0}+\sum_{n=1}^{\infty} A_{n} \exp \left(-\frac{v_{d} n^{2} \pi^{2} t}{2}\right) \cos n \pi x
$$

with Fourier coefficients at $t=0$ as

$$
\begin{gathered}
A_{0}=\int_{0}^{1} \exp \left(-\frac{1}{v_{d}} \int_{0}^{x} w_{0}(\xi) d \xi\right) d x, \\
A_{n}=2 \int_{0}^{1} \exp \left(-\frac{1}{v_{d}} \int_{0}^{x} w_{0}(\xi) d \xi\right) \cos (n \pi x) d x,
\end{gathered}
$$

where $w_{0}(\xi)=w(\xi, 0)$.

Hence, the exact solution of (1.1) is given by

$$
w(x, t)=\pi v_{d}\left(\frac{\sum_{n=1}^{\infty} A_{n} \exp \left(-v_{d} n^{2} \pi^{2} t / 2\right) n \sin n \pi x}{A_{0}+\sum_{n=1}^{\infty} A_{n} \exp \left(-v_{d} n^{2} \pi^{2} t / 2\right) \cos n \pi x}\right) .
$$

\subsection{LSDFECT Rule for Linear Parabolic Equation with Neumann Boundary Condition}

We consider the application of LSDFECT rule for the time integration of diffusion equation (4.2).

As a first step, replacing the spatial derivative by the central second-order finite difference formula, we obtain the resulting semidiscretization as follows. 


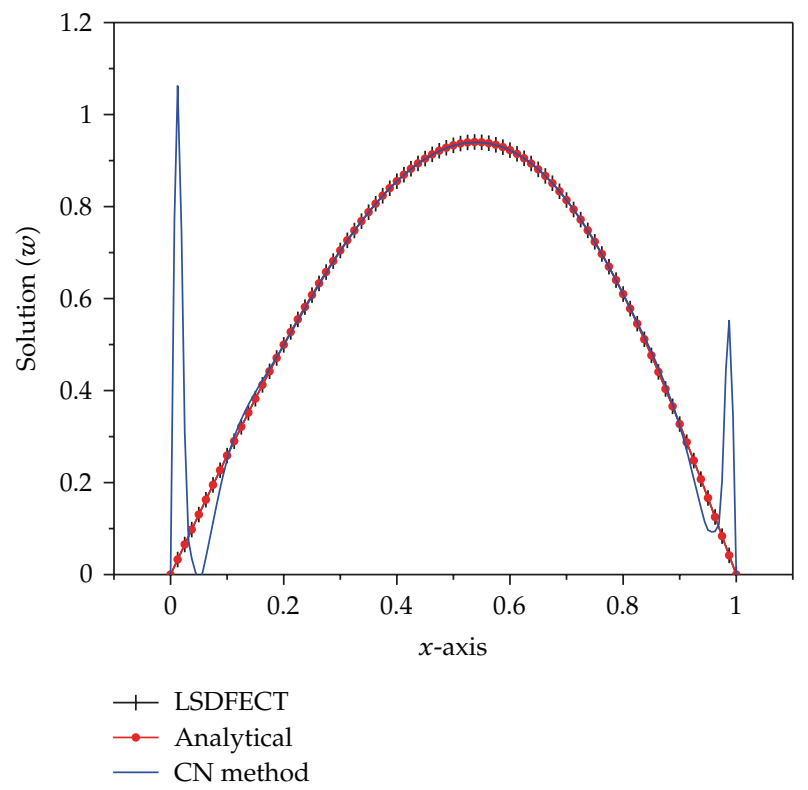

Figure 1: Comparison of the numerical solution by our method (LSDFECT), CN method, and exact solution of Problem 1 at different space points for $N=80, v_{d}=2$, and $k=0.01$ at $T=0.1$.

For a positive integer $N$, define the spatial grid $x_{i}=i h, i=0,1, \ldots, N$, where $h=1 / N$. For a positive integer $M$, define the temporal grid $t_{j}=j k, j=0,1, \ldots, M$. Now set $\phi_{i}(t)=$ $\phi\left(x_{i}, t\right)$ and so forth. Let

$$
\Phi(t)=\left[\phi_{0}(t), \phi_{1}(t), \ldots, \phi_{N}(t)\right]^{T},
$$

and $J$ is a $(N+1) \times(N+1)$ matrix given by

$$
J=\left(\begin{array}{ccccccc}
2 & -2 & 0 & 0 & \cdots & 0 & 0 \\
-1 & 2 & -1 & 0 & \cdots & 0 & 0 \\
0 & -1 & 2 & -1 & \cdots & 0 & 0 \\
\vdots & \vdots & \ddots & \ddots & \ddots & \ddots & \vdots \\
0 & 0 & 0 & -1 & 2 & -1 & 0 \\
0 & 0 & 0 & 0 & -1 & 2 & -1 \\
0 & 0 & 0 & 0 & 0 & -2 & 2
\end{array}\right) .
$$

The semidiscretization of (4.2) with the Neumann boundary conditions

$$
\phi_{x}(0, t)=0=\phi_{x}(1, t)
$$




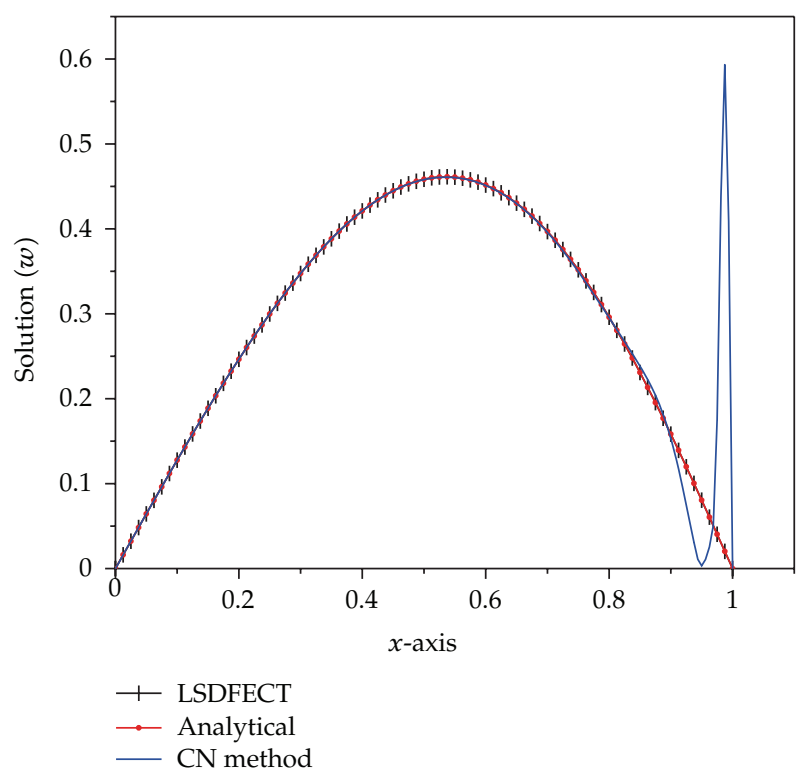

Figure 2: Comparison of the numerical solution by our method (LSDFECT), CN method, and exact solution of Problem 2 at different space points for $N=80, v_{d}=2$, and $k=0.01$ at $T=0.1$.

can be written as follows:

$$
\frac{\partial \Phi(t)}{\partial t}=-\frac{v_{d}}{2 h^{2}} J \Phi(t)
$$

where $\boldsymbol{\Phi}(0)=\left[f\left(x_{0}\right), f\left(x_{1}\right), \ldots, f\left(x_{N}\right)\right]$. Now setting $\rho=v_{d} k / 2 h^{2}$ and applying LSDFECT rule for the time integration of (4.11), we get

$$
\boldsymbol{\Phi}_{j+1}=\boldsymbol{\Phi}_{j}-\frac{1}{36} \rho J\left[3\left(\boldsymbol{\Phi}_{j}+\boldsymbol{\Phi}_{j+1}\right)+11\left(\overline{\mathbf{\Phi}}_{j+1 / 3}+\overline{\mathbf{\Phi}}_{j+2 / 3}\right)+4\left(\overline{\mathbf{\Phi}}_{j+1 / 6}+\overline{\mathbf{\Phi}}_{j+5 / 6}\right)\right]
$$

where

$$
\begin{gathered}
\overline{\boldsymbol{\Phi}}_{j+1 / 3}=\frac{1}{9}\left[4 \boldsymbol{\Phi}_{j}+(5 I+2 \rho J) \boldsymbol{\Phi}_{j+1}\right], \\
\overline{\mathbf{\Phi}}_{j+2 / 3}=\frac{1}{9}\left[\boldsymbol{\Phi}_{j}+(8 I+2 \rho J) \boldsymbol{\Phi}_{j+1}\right], \\
\overline{\mathbf{\Phi}}_{j+1 / 6}=\frac{1}{36}\left[23 \boldsymbol{\Phi}_{j}+(13 I+7 \rho J) \boldsymbol{\Phi}_{j+1}\right], \\
\overline{\mathbf{\Phi}}_{j+5 / 6}=\frac{1}{12}\left[\boldsymbol{\Phi}_{j}+(11 I+\rho J) \boldsymbol{\Phi}_{j+1}\right],
\end{gathered}
$$




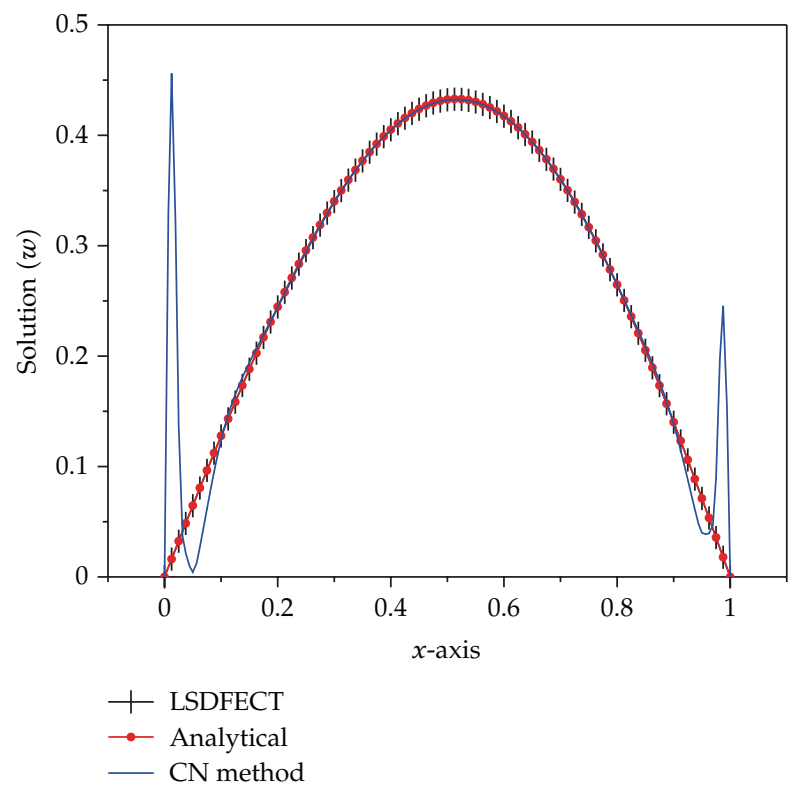

Figure 3: Comparison of the numerical solution by our method (LSDFECT), CN method, and exact solution of Problem 3 at different space points for $N=80, v_{d}=2$, and $k=0.01$ at $T=0.1$.

and $I$ is a $(N+1) \times(N+1)$ identity matrix. Using (4.12) to (4.13), we deduce

$$
\left(I+\frac{2}{3} \rho J+\frac{1}{6} \rho^{2} J^{2}\right) \overline{\mathbf{\Phi}}_{j+1}=\left(I-\frac{1}{3} \rho J\right) \overline{\mathbf{\Phi}}_{j}
$$

which is the LSDFECT rule for the linearized Burgers' equation, which is the same as the $L$-stable scheme developed by Chawla and Evans [7] and later by Pandey et al. [8].

\subsection{Unconditional Stability}

We write (4.14) in the following form:

$$
\overline{\mathbf{\Phi}}_{j+1}=Q \bar{\Phi}_{j}, \quad j=0,1,2 \ldots,
$$

where

$$
Q=\left(I+\frac{2}{3} \rho J+\frac{1}{6} \rho^{2} J^{2}\right)^{-1}\left(I-\frac{1}{3} \rho J\right)
$$

Using the Gershgorin Circle Theorem, one can prove that $J$ has positive eigenvalues. Then, it is easy to see that all the eigenvalues of $Q$ are inside unit circle. Thus, LSDFECT rule is an unconditionally stable method for all $\rho>0$. 


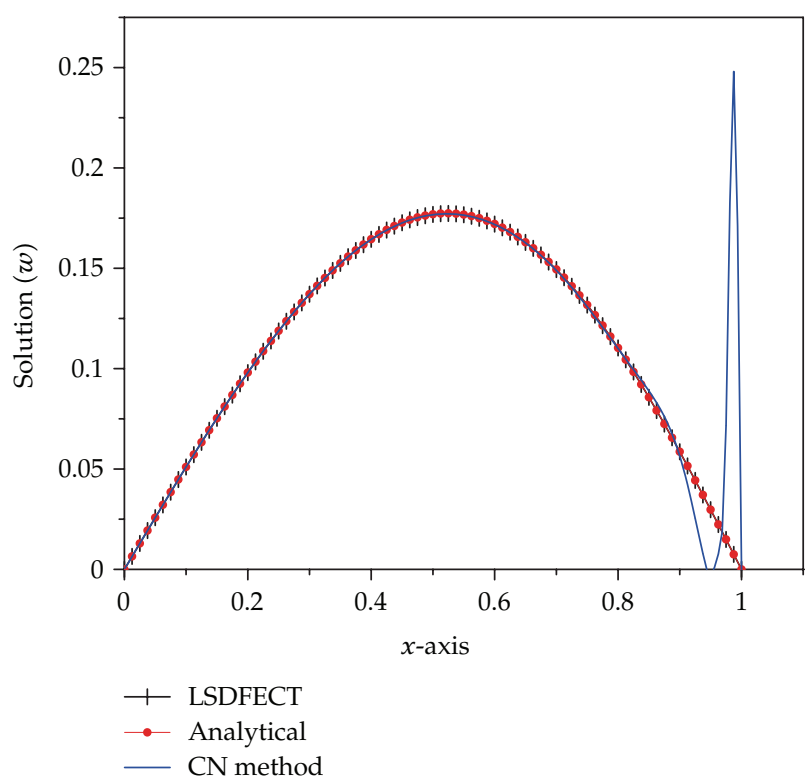

Figure 4: Comparison of the numerical solution by our method (LSDFECT), CN method, and exact solution of Problem 4 at different space points for $N=80, v_{d}=2$, and $k=0.01$ at $T=0.1$.

\section{Numerical Illustrations}

To illustrate the computational performance of the LSDFECT rule and to compare its performance with $\mathrm{CN}$, we consider five examples. First four examples contain inconsistent initial and boundary conditions. It is easily seen from Figures 1, 2, 3, and 4 that LSDFECT rule gives us stable and accurate result whereas $\mathrm{CN}$ method has large oscillations near boundary points.

In Problem 5, we have considered consistent initial and boundary conditions. We have calculated solutions for $h=0.1$ and $k=4.0$ for $v_{d}=0.02$ for time $T=80$. We observe that, since time step size $k>1 / \pi, \mathrm{CN}$ method gives large oscillations which is unacceptable, while LSDFECT rule gives quite stable and accurate solutions (see Figure 5).

\subsection{Problem 1}

Consider (1.1) with the boundary conditions (1.3) and initial condition

$$
w(x, 0)=2, \quad 0<x<1 .
$$

The exact solution is given by (4.7), where

$$
A_{0}=\int_{0}^{1} \exp \left(\frac{-2 x}{v_{d}}\right) d x, \quad A_{n}=2 \int_{0}^{1} \exp \left(\frac{-2 x}{v_{d}}\right) \cos (n \pi x) d x
$$




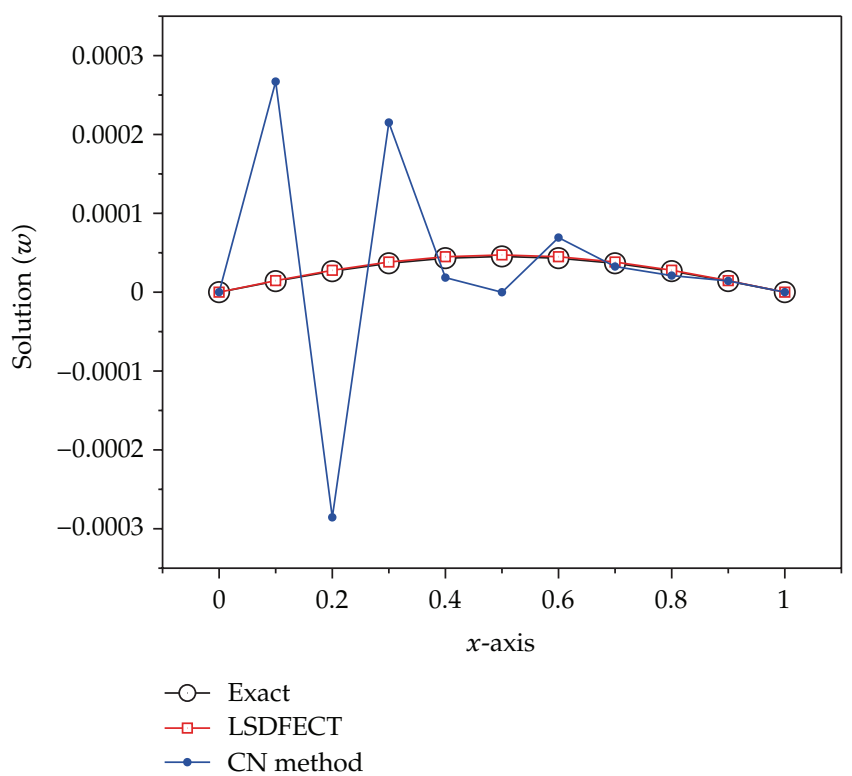

Figure 5: Comparison of the numerical solution by our method (LSDFECT), CN method, and exact solution of Problem 5 at different space points for $h=0.1, v_{d}=0.02$, and $k=4$ at $T=80$.

\subsection{Problem 2}

Consider (1.1) with the boundary conditions (1.3) and initial condition

$$
w(x, 0)=2 x, \quad 0<x<1
$$

The exact solution is given by (4.7), where

$$
A_{0}=\int_{0}^{1} \exp \left(\frac{-x^{2}}{v_{d}}\right) d x, \quad A_{n}=2 \int_{0}^{1} \exp \left(\frac{-x^{2}}{v_{d}}\right) \cos (n \pi x) d x
$$

\subsection{Problem 3}

Consider (1.1) with the boundary conditions (1.3) and initial condition

$$
w(x, 0)=\cos \frac{\pi}{4} x, \quad 0<x<1 .
$$

The exact solution is given by (4.7), where

$$
A_{0}=\int_{0}^{1} \exp \left(-\frac{4}{\pi v_{d}} \sin \frac{\pi}{4} x\right) d x, \quad A_{n}=2 \int_{0}^{1} \exp \left(-\frac{4}{\pi v_{d}} \sin \frac{\pi}{4} x\right) \cos (n \pi x) d x
$$




\subsection{Problem 4}

Consider (1.1) with the boundary conditions (1.3) and initial condition

$$
w(x, 0)=\sin \frac{\pi}{4} x, \quad 0<x<1 .
$$

The exact solution is given by (4.7), where

$$
A_{0}=\int_{0}^{1} \exp \left(\frac{4}{\pi v_{d}}\left(\cos \frac{\pi}{4} x-1\right)\right) d x, \quad A_{n}=2 \int_{0}^{1} \exp \left(\frac{4}{\pi v_{d}}\left(\cos \frac{\pi}{4} x-1\right)\right) \cos (n \pi x) d x
$$

\subsection{Problem 5}

Consider (1.1) with the boundary conditions (1.3) and initial condition

$$
w(x, 0)=\sin \pi x, \quad 0<x<1 .
$$

The exact solution is given by (4.7), where

$$
\begin{gathered}
A_{0}=\int_{0}^{1} \exp \left(\frac{-1}{\pi v_{d}}(1-\cos \pi x)\right) d x \\
A_{n}=2 \int_{0}^{1} \exp \left(\frac{-1}{\pi v_{d}}(1-\cos \pi x)\right) \cos n \pi x d x
\end{gathered}
$$

\section{Conclusion}

Using derivative-free error-corrected trapezoidal rule, suitable average approximation, and explicit backward Euler formula, we derive third-order integration scheme in time which is $L$-stable. The present method provides accurate and stable results whenever inconsistencies are present.

\section{References}

[1] J. Rakshit and P. Kumar, Derivative free correction in trapezoidal rule [M.S. thesis], IIT Kharagpur, 2007.

[2] J. Crank and P. Nicolson, "A practical method for numerical evaluation of solutions of partial differential equations of the heat-conduction type," Mathematical Proceedings of the Cambridge Philosophical Society, vol. 43, no. 1, pp. 50-67, 1947.

[3] R. D. Richtmyer and K. W. Morton, Difference Methods for Initial-Value Problems, Interscience Tracts in Pure and Applied Mathematics, Wiley-Interscience, New York, NY, USA, 2nd edition, 1967.

[4] J. D. Lawson and J. L. Morris, "The extrapolation of first order methods for parabolic partial differential equations I," SIAM Journal on Numerical Analysis, vol. 15, no. 6, pp. 1212-1224, 1978.

[5] M. M. Chawla, M. A. Al-Zanaidi, and D. J. Evans, "Generalized trapezoidal formulas for parabolic equations," International Journal of Computer Mathematics, vol. 70, no. 3, pp. 429-443, 1999.

[6] M. M. Chawla, M. A. Al-Zanaidi, and M. S. Al-Sahhar, "Stabilized fourth order extended methods for the numerical solution of ODEs," International Journal of Computer Mathematics, vol. 52, pp. 99-107, 1994. 
[7] M. M. Chawla and D. J. Evans, "A new L-stable Simpson-type rule for the diffusion equation," International Journal of Computer Mathematics, vol. 82, no. 5, pp. 601-607, 2005.

[8] K. Pandey, L. Verma, and A. K. Verma, "L-stable Simpson's $3 / 8$ rule and Burgers' equation," Applied Mathematics and Computation, vol. 218, no. 4, pp. 1342-1352, 2011.

[9] M. M. Chawla, M. A. Al-Zanaidi, and D. J. Evans, "A class of generalized trapezoidal formulas for the numerical integration of $y^{\prime}=f(x, y)$," International Journal of Computer Mathematics, vol. 62, no. 1-2, pp. 131-142, 1996.

[10] E. Hopf, "The partial differential equation $u_{t}+u u_{x}=\mu u_{x x}$," Communications on Pure and Applied Mathematics, vol. 3, pp. 201-230, 1950.

[11] J. D. Cole, "On a quasi-linear parabolic equation occurring in aerodynamics," Quarterly of Applied Mathematics, vol. 9, pp. 225-236, 1951. 


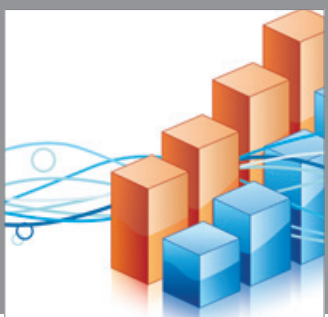

Advances in

Operations Research

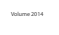

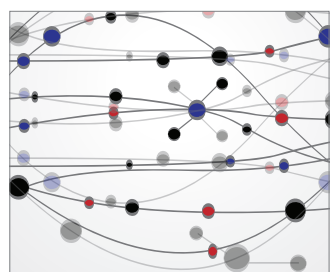

\section{The Scientific} World Journal
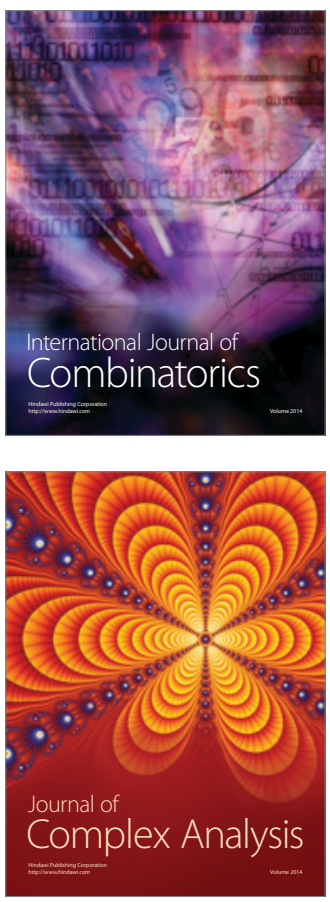

International Journal of

Mathematics and

Mathematical

Sciences
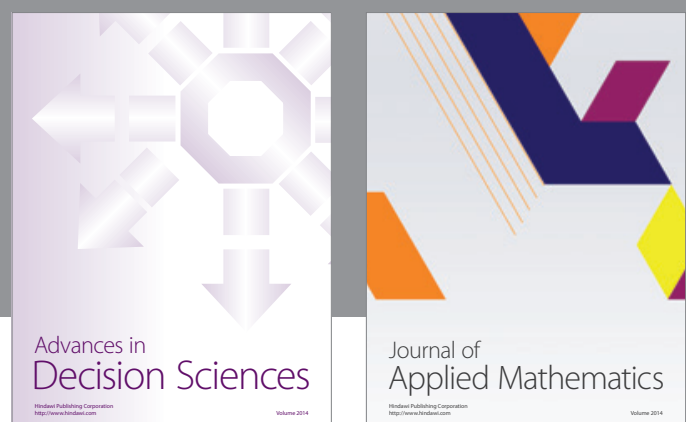

Journal of

Applied Mathematics
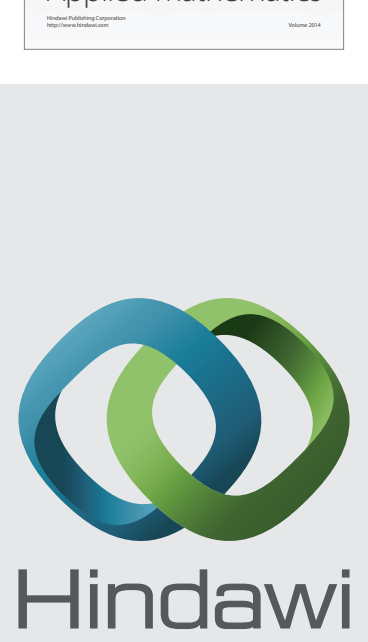

Submit your manuscripts at http://www.hindawi.com
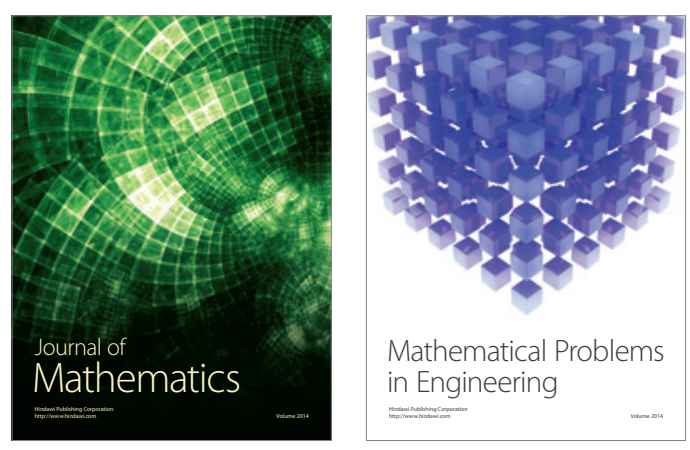

Mathematical Problems in Engineering
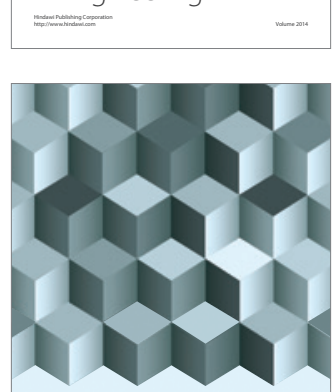

Journal of

Function Spaces
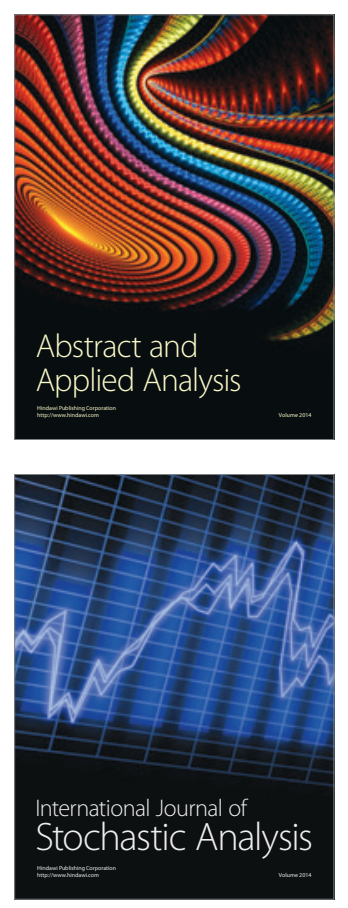

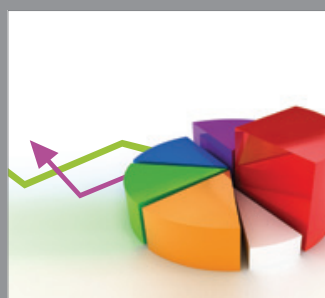

ournal of

Probability and Statistics

Promensencen
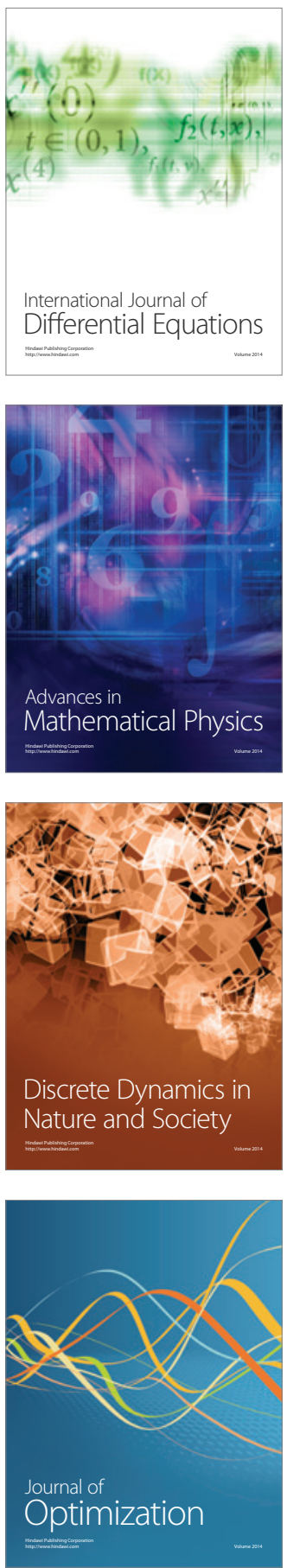